\title{
Genotype Distribution of Human Papillomaviruses in Japanese Women with Abnormal Cervical Cytology
}

\author{
Kazunari Kondo ${ }^{1}$, Asami Uenoyama ${ }^{1}$, Ryo Kitagawa ${ }^{1}$, Hajime Tsunoda ${ }^{1}$, Rika Kusumoto-Matsuo ${ }^{2}$, \\ Seiichiro Mori ${ }^{2}$, Yoshiyuki Ishii ${ }^{2}$, Takamasa Takeuchi ${ }^{2}$, Tadahito Kanda ${ }^{3}$ and Iwao Kukimoto ${ }^{* 2}$ \\ ${ }^{I}$ NTT Medical Center Tokyo, 5-9-22 Higashi-gotanda, Shinagawa-ku, Tokyo 141-8625, Japan \\ ${ }^{2}$ WHO HPV LabNet Regional Reference Laboratory, WHO Western Pacific Region, Pathogen Genomics Center, \\ National Institute of Infectious Diseases, 4-7-1 Gakuen, Musashi-murayama, Tokyo 208-0011, Japan \\ ${ }^{3}$ Center of Research Network for Infectious Diseases, Riken, 1-101 Kanda-jinbocho, Chiyoda-ku, Tokyo 101-0051, \\ Japan
}

\begin{abstract}
We report the prevalence and genotype distribution of human papillomaviruses (HPVs) among Japanese women with abnormal cervical cytology using the PGMY-CHUV assay, one of PGMY-PCR-based lineblot assays that was validated and shown to be suitable for the detection of multiple HPV types in a specimen with minimum bias. Total DNA was extracted from cervical exfoliated cells collected from 326 outpatients with abnormal Pap smears. Overall, 307 specimens (94\%) were HPV-positive, $30 \%$ of which contained multiple genotypes. The prevalence of HPV DNA was $83 \%$ (49/59 samples) in atypical squamous cells of undetermined significance (ASC-US); $91 \%(20 / 22$ samples) in atypical squamous cells, cannot exclude high-grade squamous intraepithelial lesion (ASC-H); 97\% (130/134 samples) in low-grade squamous intraepithelial lesion (LSIL); and 99\% (85/86 samples) in high-grade squamous intraepithelial lesion (HSIL). Three most frequent HPV types detected in HSIL were HPV16 (36\%), HPV52 (24\%), and HPV58 (14\%). Our results suggest that multiple HPV infections are more prevalent in Japanese women than previously reported, and confirm that HPV52 and 58 are more dominant in their cervical precancerous lesions when compared to those reported in Western countries.
\end{abstract}

Keywords: Human papillomavirus, HPV genotyping, cervical cancer, Pap smear, abnormal cytology, HPV vaccine.

\section{INTRODUCTION}

Human papillomaviruses (HPVs) are the causative agents of cervical cancer, cervical intraepithelial neoplasia, and other anogenital cancers [1]. Among more than $100 \mathrm{HPV}$ types so far identified, nearly 40 types infecting the anogenital mucosa are classified as either low- or high-risk types on the basis of their oncogenic potential [2]. A previous large-scale epidemiological study identified 15 HPV types, HPV16, 18, 31, 33, 35, 39, 45, 51, 52, 56, 58, $59,68,73$, and 82 , which are closely linked to the development of cervical cancer [3], with approximately $70 \%$ of cervical cancer cases worldwide attributed to HPV16 and 18 [4].

Two prophylactic HPV vaccines, one quadrivalent vaccine directed against HPV6, 11, 16, and $18\left[\right.$ Gardasi $^{\mathbb{B}}{ }^{1}$ (Merck and Co., Whitehouse Station, NJ, USA)], and one bivalent vaccine against HPV16 and 18 [Cervarix ${ }^{\circledR}$ (GlaxoSmithKline Biologicals, Rixensart, Belgium)], are now being introduced worldwide. Both vaccines exhibit a high efficacy for preventing cervical precancerous lesions caused by vaccine-targeted HPV types in clinical trials for

*Address correspondence to this author at the Pathogen Genomics Center, National Institute of Infectious Diseases, 4-7-1 Gakuen, Musashimurayama, Tokyo 208-0011, Japan; Tel: +81 42561 0771; Fax: +81 42567 5632; E-mail: ikuki@nih.go.jp
HPV-uninfected women $[5,6]$. In Japan, the bivalent vaccine was first approved for use in 2009, followed by the quadrivalent vaccine in 2011, both being introduced with the expectation of reducing cervical cancer cases. However, previous epidemiological studies performed in Japanese women have shown variation in the proportion of HPV16 and 18 in cervical cancer, ranging from 50 to $70 \%$ [7-9], which makes it difficult to predict the real impact of the HPV vaccination on cervical cancer prevention in the Japanese population.

Because currently available HPV vaccines are thought to be effective only for the vaccine-targeted types, precise determination of the HPV genotype distribution in cervical cancer and its precancerous lesions is needed for assessment of the vaccine efficacy and planning of future vaccination strategies. In 2006, the World Health Organization (WHO) established the HPV laboratory network (LabNet) with the aim of harmonizing and standardizing laboratory testing procedures to promote consistent laboratory evaluation of HPV-related disease burden and monitoring of the performance of HPV vaccines. Through a series of collaborative studies for standardizing HPV genotyping assays, the LabNet has recently described a PGMY-lineblot assay (PGMY-CHUV [10]) as a reliable, low-cost HPV genotyping method [11,12]. The PGMY-CHUV assay consists of PCR with biotinylated generic PGMY09/11 
primers to amplify the HPV L1 region and subsequent reverse-blotting hybridization with type-specific DNA probes, and is suitable to detect multiple HPV types in a specimen with minimum bias $[10,13]$. Most studies on the HPV genotype distribution in Japanese women, however, have relied on PCR of HPV DNA using a limited pair of L1 consensus primers, such as LC1/LC2 [7,14-20], which is less sensitive to detect multiple-genotypes infection compared to other PCR assays using type-specific primers, which might have resulted in less confident data on HPV prevalence in Japanese women.

In this study we examined the prevalence and distribution of HPV genotypes among Japanese women with abnormal Pap smears by using the PGMY-CHUV assay. The data indicate that multiple HPV infections are more prevalent in Japanese women than previously reported, and confirm that HPV52 and 58 are more dominant in cervical precancerous lesions of Japanese women than in those of Western countries' women.

\section{MATERIALS AND METHODOLOGY}

\section{Study Population and Specimen Collection}

From November 2009 to May 2011, we enrolled 1088 women who visited NTT Medical Center Tokyo, a regional medical center in Tokyo, as outpatients having some sort of symptoms or referred by primary care physicians for further examination. The women were subjected to the Papanicolaou (Pap) smear test, leading to diagnoses of $762(70 \%)$ negative for intraepithelial lesion or malignancy (NILM) and 326 $(30 \%)$ abnormal cytology cases, based on the criteria of the Bethesda System 2001 (Fig. 1). Together with sampling Pap smears, cervical exfoliated cells were collected in Thinprep ${ }^{B}$

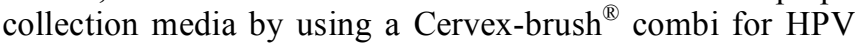
genotyping. The women with abnormal cytology were further examined by colposcopy, and tissue specimens obtained by punch biopsy were histologically diagnosed. Among 86 HSIL cases, 53 cases were treated by cervical conization, and excised tissues were finally histologically diagnosed as 1 CIN1, 9 CIN2, and 42 CIN3 cases (Fig. 1). All ICC cases diagnosed by the Pap test were finally histologically confirmed by tissue biopsy. The study protocol was approved by the Ethics Committee at NTT Medical Center Tokyo, and written informed consent for study participation was obtained from each patient.

\section{HPV DNA Detection and Genotyping}

Total DNA was extracted from a $200-\mu \mathrm{L}$ aliquot of the suspended cell samples by using the QIAamp DNA Blood Mini Kit (Qiagen), resulting in the final elution volume of $100 \mu \mathrm{L}$ Tris-EDTA buffer. Cross-contamination between samples during DNA extraction was evaluated by simultaneous extraction from only a collection medium. A 3$\mu \mathrm{L}$ aliquot of the purified DNA was then used for PCR amplification with AmpliTaq Gold ${ }^{\circledR}$ polymerase (GE Healthcare Bio-Sciences), biotinylated PGMY09/11 primers, to amplify the L1 DNA of $>40 \mathrm{HPV}$ genotypes, and biotinylated HLA primers, to amplify cellular HLA DNA. Positive control $(0.1 \mathrm{pg} / \mathrm{mL}$ of HPV16 DNA as a plasmid) and negative control $\left(\mathrm{dH}_{2} \mathrm{O}\right)$ were included in every $50 \mathrm{PCR}$ reactions to verify the sensitivity of PCR and monitor contamination of HPV DNA in reagents. The PCR products were run on $1.5 \%$ agarose gels to assign the positivity of HPV DNA amplification and to confirm the integrity of the extracted DNA as amplification of HLA DNA. Reverse blotting hybridization was done as described in the Human Papillomavirus Laboratory Manual published by the WHO [11]. Briefly, 15- $\mu \mathrm{L}$ denatured PCR products were allowed to hybridize with oligonucleotide probes specific for $31 \mathrm{HPV}$ types (HPV6, 11, 16, 18, 26, 31, 33, 34, 35, 39, 40, 42, 44, $45,51,52,53,54,55,56,57,58,59,66,68,69,70,73,82$, 83 , and 84) immobilized on a Biodyne $\mathrm{C}$ membrane (Pall corporation) using a Miniblotter MN45 (Immunetics, Cambridge, MA, USA). The hybridized DNA was detected using streptavidin-HRP (GE Healthcare Bio- Sciences, Piscataway, NJ, USA) and ECL detection reagent (GE Healthcare Bio-Sciences). After reading positive signals, PCR-positive but hybridization-negative samples were

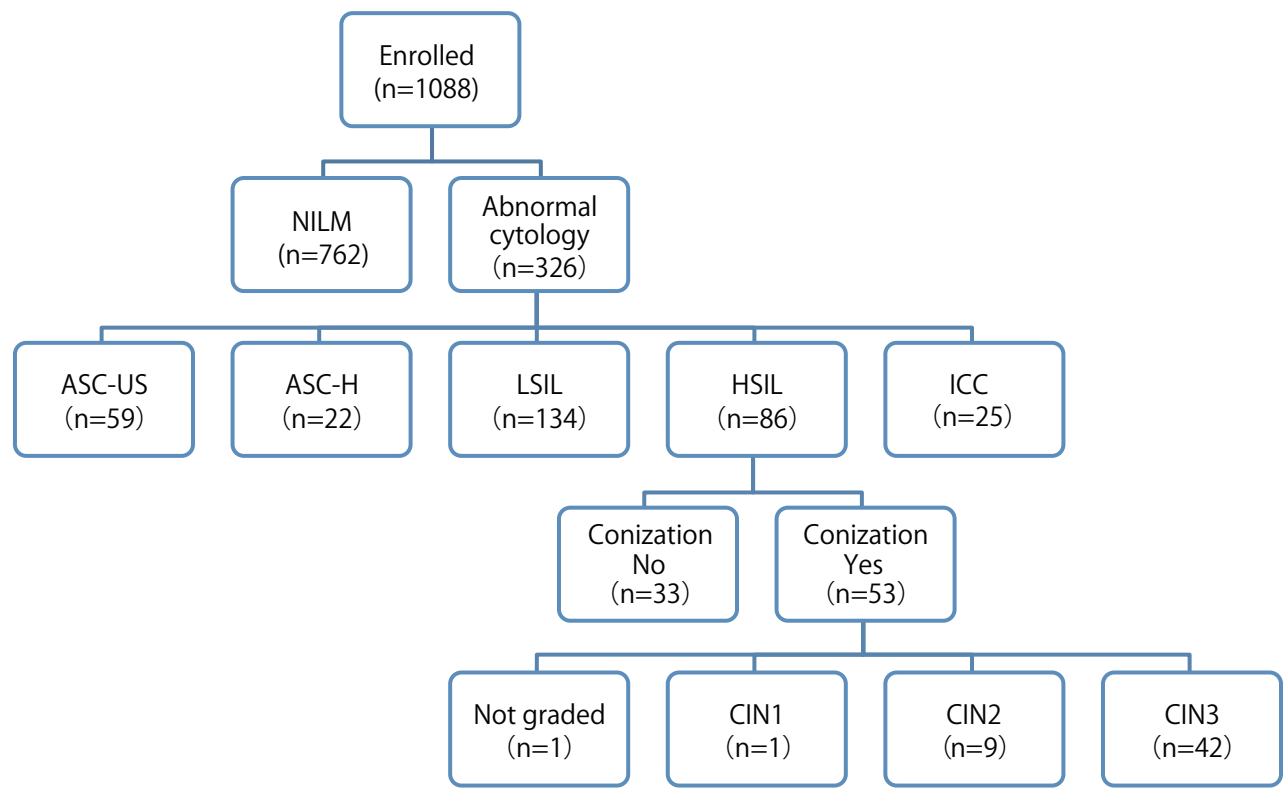

Fig. (1). Overview of study population. $\mathrm{n}$ indicates numbers of patients. 
further subjected to direct sequencing of the PGMY PCR products with the PGMY11 primer. Our typing capability using the PGMY-CHUV assay was approved as proficient in the HPV DNA proficiency panel studies conducted annually by the WHO HPV LabNet [12].

\section{Statistical Analysis}

To test differences between population proportions, Pearson's chi-squared test with Yates' continuity correction was performed using $\mathrm{R}$ version 2.11.1. Two-sided $P$ values were calculated and considered to be significant at less than 0.05 . Confidence intervals (CI) at $95 \%$ level for population proportions were calculated using $\mathrm{R}$ based on the assumption of the $F$ distribution.

\section{RESULTS}

The overall prevalence of HPV DNA among 326 women with abnormal Pap smears (age 17 to 84 , mean age \pm standard deviation: $38.9 \pm 10$ years) was $94.2 \%(307 / 326)$ (Table 1). The study subjects were stratified based on their cytological status. The mean age \pm standard deviation in each status was as follows: atypical squamous cells of undetermined significance (ASC-US), $38.3 \pm 9.4$ years; atypical squamous cells, cannot exclude high-grade squamous intraepithelial lesion (ASC-H), $38.8 \pm 9.8$ years; low-grade squamous intraepithelial lesion (LSIL), $37.4 \pm 10$ years; high-grade squamous intraepithelial lesion (HSIL), $38.3 \pm 8.9$ years; and invasive cervical cancer (ICC), $52.0 \pm$ 14 years. The detection rate of HPV DNA in each cytological group was as follows: ASC-US, 83.1\% (49/59); ASC-H, 90.9\% (20/22); LSIL, 97.0\% (130/134); HSIL, 98.8\% (85/86); and ICC, 92.0\% (23/25). Meanwhile, among 762 NILM cases, the prevalence of HPV DNA in 173 pregnant women without cervical abnormalities (mean age \pm standard deviation: $31.8 \pm 4.8$ years) was $24.3 \%(42 / 173)$ (data not shown).

Overall, among the 307 HPV-positive women with cervical abnormalities, HPV16 (21.2\%), HPV52 (20.2\%), and HPV58 (14.7\%) were predominantly detected, which was followed by HPV56 (8.3\%), HPV51 (7.4\%), and HPV31 (7.4\%) (Table 2). The genotype distribution varied with the status of lesion abnormalities: in ASC-US, HPV52 (20.3\%), HPV16 (15.3\%), HPV58 (13.6\%), and HPV53 (10.2\%); in ASC-H, HPV52 (40.9\%), HPV16 (22.7\%), HPV58 (18.2\%) and HPV31 and HPV51 (13.6\%); in LSIL, HPV58 (17.2\%), HPV52 (16.4\%), HPV56 (14.9\%), HPV16 (10.4\%), and HPV66 (9.7\%); in HSIL, HPV16 (36.0\%), HPV52 (24.4\%), HPV58 (14.0\%), HPV31 (11.6\%), and HPV18 and HPV51 $(9.3 \%)$. Comparison of the detection rate of high-risk types between LSIL and HSIL showed that HPV16 and 31 were significantly more prevalent in HSIL $(p=0.00001$ for HPV16, and 0.046 for HPV31), while the reverse was observed for HPV56 and 66 ( $p=0.013$ for HPV56, and 0.025 for HPV66) (Fig. 2a). Sequencing of PCR-positive and hybridization-negative samples ( $\mathrm{X}$ in Table 2 ) identified HPV types not included in the probes on the typing membrane: HPV61, 62, 67, 71, 74, 86, 87, and 90, all of which belong to the low-risk genotype.

Multiple HPV genotypes were detected in $29.6 \%$ of total HPV-positive subjects (91/307) (Table 3). The detection rate of multiple HPV genotypes in each cytological group was as follows: ASC-US, 24.5\% (12/49); ASC-H, 40.0\% (8/20); LSIL, 32.3\% (42/130); HSIL, 31.8\% (27/85); and ICC, $8.7 \%$ $(2 / 23)$. Among the 91 subjects of multiple infections of any HPVs, the detection rate of multiple high-risk types was 84.6\% (77/91): ASC-US, 66.7\% (8/12); ASC-H, 100\% (8/8); LSIL, 83.3\% (35/42); HSIL, 88.9\% (24/27); and ICC, 100\% $(2 / 2)$. The 2 cases of multiple infections in ICC were coinfections of HPV16 and 53, and of HPV31 and 53. Overall, the most frequent combination of multiple infections was coinfection of HPV16 and 52 (9.9\%), followed by co-infection of HPV52 and $58(9.9 \%)$. As shown in Fig. (3), the proportion of HPV16 and/or 18 infections without other high-risk HPVs was significantly higher in HSIL than in LSIL $(p=0.0002)$, and was further higher in ICC than in HSIL $(p=0.045)$. Conversely, the proportion of high-risk HPV infections other than HPV16/18 was significantly lower in HSIL than in LSIL $(p=0.026)$, and was slightly lower in ICC compared to HSIL (but not statistically significant). The proportion of multiple infections of HPV16 and/or 18 with other high-risk HPVs was highest in HSIL $(15.1 \%)$, but was lowest in ICC (4.0\%). Among the 307 HPV-positive women, high- and low-risk genotypes were detected in $281(91.5 \%)$ and $46(15.0 \%)$ women, respectively, and $26(8.5 \%)$ women were infected with both types.

In the 86 subjects of HSIL, 42 women (mean age \pm standard deviation: $38.6 \pm 6.1$ years) were histologically diagnosed as CIN3 by cervical cone biopsy (see Fig. 1). The detection rate of high-risk HPV DNA in the CIN3 cases was $100 \%, 33.3 \%$ of which corresponded to multiple infections (14/42). Three most frequent genotypes in the CIN3 cases were HPV16 (45.2\%), HPV58 (19.0\%), and HPV52 (14.3\%) (Fig. 2b). Among the 25 cases of ICC, 20 cases were histologically diagnosed as squamous cell carcinoma (SCC), whereas the remaining 5 cases were determined as adenocarcinoma. In SCC, HPV16 and 18 were detected in $40.0 \%$ and $10.0 \%$, respectively (Fig. 2b). In the 5 cases of

Table 1. Prevalence of HPV DNA in Japanese Women with Abnormal Cervical Cytology

\begin{tabular}{|c|c|c|c|c|c|c|}
\hline & Total & ASC-US & ASC-H & LSIL & HSIL ICC & 86 \\
\hline \hline $\mathrm{N}^{*}$ & 326 & 59 & 22 & 134 & 130 & 85 \\
\hline $\mathrm{HPV}(+)^{*}$ & 307 & 49 & 20 & 97.0 & 98.8 & 92.0 \\
\hline$\%^{* *}$ & 94.2 & 83.1 & 90.9 & $37.4 \pm 10$ & $38.3 \pm 8.9$ & $52.0 \pm 14$ \\
\hline $\mathrm{Age}^{* * *}$ & $38.9 \pm 10$ & $38.3 \pm 9.4$ & $38.8 \pm 9.8$ & & \\
\hline
\end{tabular}

*, Numbers of patients

**, Percentages of HPV-positive patients relative to the total number of patients in each cytological group.

$* * *$, Mean age \pm standard deviation. 
Table 2. HPV Type Prevalence in Japanese Women with Abnormal Cervical Cytology

\begin{tabular}{|c|c|c|c|c|c|c|c|c|c|c|c|c|}
\hline \multirow{2}{*}{ HPV Types } & \multicolumn{2}{|c|}{ Total $(N=326)$} & \multicolumn{2}{|c|}{ ASC-US $(N=59)$} & \multicolumn{2}{|c|}{ ASC-H $(N=22)$} & \multicolumn{2}{|c|}{ LSIL $(N=134)$} & \multicolumn{2}{|c|}{ HSIL $(N=86)$} & \multicolumn{2}{|c|}{$\operatorname{ICC}(\mathrm{N}=25)$} \\
\hline & $\mathbf{n}^{*}$ & $\%^{* *}$ & $\mathbf{n}^{*}$ & $\%^{* *}$ & $\mathbf{n}^{*}$ & $\%{ }^{* *}$ & $\mathrm{n}^{*}$ & $\%^{* *}$ & $\mathbf{n}^{*}$ & $\%^{* * *}$ & $\mathbf{n}^{*}$ & $\%^{* *}$ \\
\hline \multicolumn{13}{|l|}{ High-Risk } \\
\hline 16 & 69 & 21.2 & 9 & 15.3 & 5 & 22.7 & 14 & 10.4 & 31 & 36.0 & 10 & 40.0 \\
\hline 18 & 23 & 7.1 & 1 & 1.7 & 1 & 4.5 & 9 & 6.7 & 8 & 9.3 & 4 & 16.0 \\
\hline 26 & 1 & 0.3 & 0 & 0 & 0 & 0 & 0 & 0 & 1 & 1.2 & 0 & 0 \\
\hline 31 & 24 & 7.4 & 3 & 5.1 & 3 & 13.6 & 5 & 3.7 & 10 & 11.6 & 3 & 12.0 \\
\hline 33 & 6 & 1.8 & 0 & 0 & 0 & 0 & 2 & 1.5 & 3 & 3.5 & 1 & 4.0 \\
\hline 35 & 6 & 1.8 & 0 & 0 & 0 & 0 & 3 & 2.2 & 3 & 3.5 & 0 & 0 \\
\hline 39 & 16 & 4.9 & 3 & 5.1 & 0 & 0 & 11 & 8.2 & 1 & 1.2 & 1 & 4.0 \\
\hline 45 & 1 & 0.3 & 0 & 0 & 0 & 0 & 0 & 0 & 0 & 0 & 1 & 4.0 \\
\hline 51 & 24 & 7.4 & 2 & 3.4 & 3 & 13.6 & 11 & 8.2 & 8 & 9.3 & 0 & 0 \\
\hline 52 & 66 & 20.2 & 12 & 20.3 & 9 & 40.9 & 22 & 16.4 & 21 & 24.4 & 2 & 8.0 \\
\hline 53 & 23 & 7.1 & 6 & 10.2 & 1 & 4.5 & 12 & 9.0 & 2 & 2.3 & 2 & 8.0 \\
\hline 56 & 27 & 8.3 & 3 & 5.1 & 1 & 4.5 & 20 & 14.9 & 3 & 3.5 & 0 & 0 \\
\hline 58 & 48 & 14.7 & 8 & 13.6 & 4 & 18.2 & 23 & 17.2 & 12 & 14.0 & 1 & 4.0 \\
\hline 59 & 10 & 3.1 & 3 & 5.1 & 1 & 4.5 & 4 & 3.0 & 2 & 2.3 & 0 & 0 \\
\hline 66 & 15 & 4.6 & 1 & 1.7 & 0 & 0 & 13 & 9.7 & 1 & 1.2 & 0 & 0 \\
\hline 68 & 5 & 1.5 & 1 & 1.7 & 0 & 0 & 2 & 1.5 & 2 & 2.3 & 0 & 0 \\
\hline 73 & 1 & 0.3 & 1 & 1.7 & 0 & 0 & 0 & 0 & 0 & 0 & 0 & 0 \\
\hline 82 & 6 & 1.8 & 1 & 1.7 & 0 & 0 & 1 & 0.7 & 4 & 4.7 & 0 & 0 \\
\hline Any $^{* * *}$ & 281 & 86.2 & 44 & 74.6 & 20 & 90.9 & 111 & 82.8 & 83 & 96.5 & 23 & 92.0 \\
\hline
\end{tabular}

Low-Risk

\begin{tabular}{|c|c|c|c|c|c|c|c|c|c|c|c|c|}
\hline 6 & 8 & 2.5 & 1 & 1.7 & 0 & 0 & 4 & 3.0 & 3 & 3.5 & 0 & 0 \\
\hline 11 & 1 & 0.3 & 1 & 1.7 & 0 & 0 & 0 & 0 & 0 & 0 & 0 & 0 \\
\hline 34 & 1 & 0.3 & 0 & 0 & 0 & 0 & 1 & 0.7 & 0 & 0 & 0 & 0 \\
\hline 40 & 0 & 0 & 0 & 0 & 0 & 0 & 0 & 0 & 0 & 0 & 0 & 0 \\
\hline 42 & 9 & 2.8 & 3 & 5.1 & 0 & 0 & 5 & 3.7 & 1 & 1.2 & 0 & 0 \\
\hline 43 & 0 & 0 & 0 & 0 & 0 & 0 & 0 & 0 & 0 & 0 & 0 & 0 \\
\hline 44 & 0 & 0 & 0 & 0 & 0 & 0 & 0 & 0 & 0 & 0 & 0 & 0 \\
\hline 54 & 6 & 1.8 & 1 & 1.7 & 0 & 0 & 5 & 3.7 & 0 & 0 & 0 & 0 \\
\hline 55 & 0 & 0 & 0 & 0 & 0 & 0 & 0 & 0 & 0 & 0 & 0 & 0 \\
\hline 57 & 0 & 0 & 0 & 0 & 0 & 0 & 0 & 0 & 0 & 0 & 0 & 0 \\
\hline 69 & 1 & 0.3 & 0 & 0 & 0 & 0 & 1 & 0.7 & 0 & 0 & 0 & 0 \\
\hline 70 & 6 & 1.8 & 2 & 3.4 & 0 & 0 & 3 & 2.2 & 1 & 1.2 & 0 & 0 \\
\hline 83 & 2 & 0.6 & 0 & 0 & 0 & 0 & 2 & 1.5 & 0 & 0 & 0 & 0 \\
\hline 84 & 2 & 0.6 & 0 & 0 & 0 & 0 & 1 & 0.7 & 1 & 1.2 & 0 & 0 \\
\hline $\mathrm{X}$ & 13 & 4.0 & 1 & 1.0 & 0 & 0 & 10 & 7.5 & 2 & 2.3 & 0 & 0 \\
\hline Any & 46 & 14.1 & 7 & 11.9 & 0 & 0 & 31 & 23.1 & 8 & 9.3 & 0 & 0 \\
\hline Overall & 307 & 94.2 & 49 & 83.1 & 20 & 90.9 & 130 & 97.0 & 85 & 98.8 & 23 & 92.0 \\
\hline
\end{tabular}

HPV genotypes are grouped based on the risk classification of Munoz et al. [3]. Type-specific prevalence includes HPVs in single or multiple infections.

*, Numbers of patients detected with each HPV.

**, Percentages relative to the total number of patients $(\mathrm{N})$ in each cytological group.

***, Numbers of patients with any high- or low-risk HPVs and percentages relative to the total number of patients $(\mathrm{N})$ in each cytological group.

adenocarcinoma, HPV16 was singly detected in 2 cases, and HPV18 was singly detected in 2 cases, whereas no HPV DNA was detected in one case.

\section{DISCUSSION}

This study provides the first data on the prevalence of HPV DNA and the distribution of HPV genotypes among 


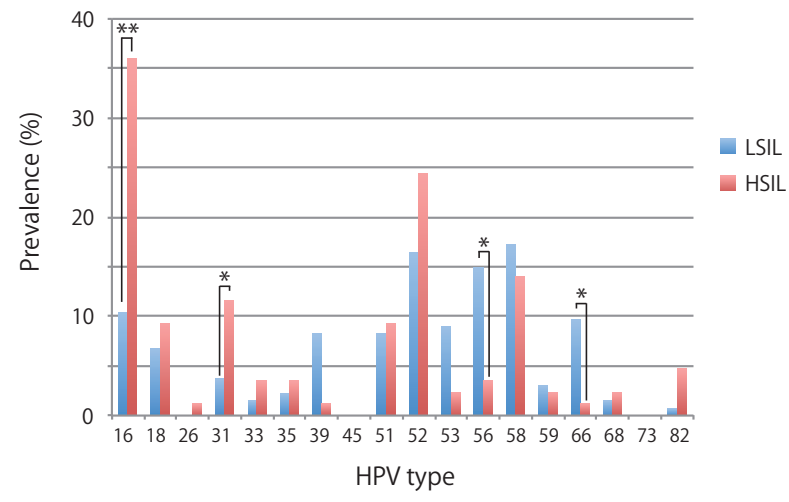

b

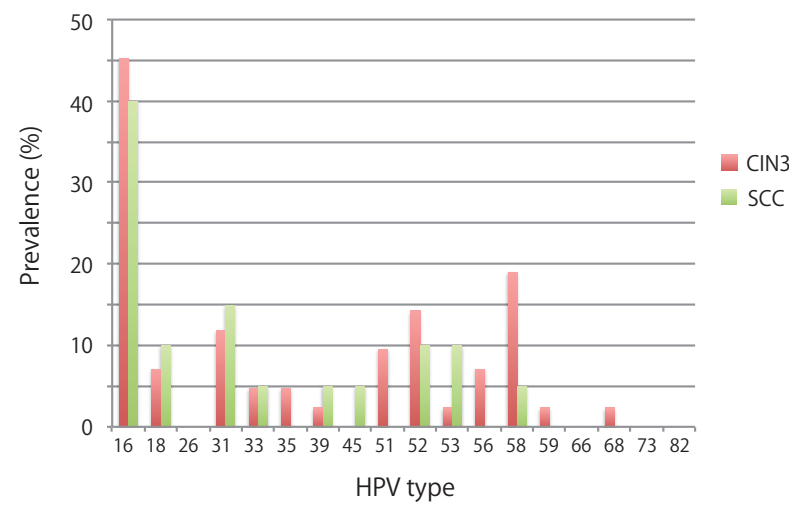

Fig. (2). Comparison of high-risk HPV type distribution in Japanese women with LSIL and HSIL (a), with CIN3 and SCC (b). * and $* *$ indicate $P<0.05$ and $P<0.001$, respectively,

Japanese women with abnormal cervical smears using a highly sensitive genotyping assay that was recently validated by the HPV LabNet. The detection rates of HPV DNA were $97.0 \%(95 \% \mathrm{CI}=93.7 \%$ to $100 \%)$ in LSIL and $98.8 \%(95 \%$ $\mathrm{CI}=92.5 \%$ to $99.2 \%)$ in HSIL, which are higher than those reported in a previous meta-analysis of studies in Japan: $79.4 \%$ in LSIL and $89.0 \%$ in HSIL [21]. With regard to multiple infections, the overall detection rate was $29.6 \%$
$(95 \%$ CI $=24.6 \%$ to $35.1 \%)$ in women with cervical abnormalities, which is also higher than a previous report: 11.3 to $18.5 \%$ in Japanese CIN patients [15]. The increased detection rate of multiple infections likely reflects a higher sensitivity of the PGMY-CHUV assay to detect multiple infections without inter-type PCR competition compared to other genotyping methods used in previous studies in Japan, such as the L1C1/L1C2 primer system [13], and/or higher performance of AmpliTaq Gold ${ }^{\circledR}$ polymerase to amplify multiple HPV DNAs with the PGMY primers compared to other PCR enzymes [22].

The positivity of HPV DNA in ASC-US (83.1\%, 95\% CI $=71.0 \%$ to $91.2 \%$ ) in this study seem to be higher than those reported in studies for cervical low-grade lesions in the US (61\%) [23] and in Sweden (63\%) [24]. This difference is likely due to the characteristics of our study population, which was not selected from primary screening programs but enrolled from outpatients mostly referred by primary care physicians due to abnormal cytology results. Furthermore, strict assignment of ASC-US cases in this study population, which is presumed to be relatively more HPV-positive than normal population, might have led to the high positivity of HPV DNA in ASC-US.

The previous meta-analysis of the HPV type prevalence in Japan showed that three most frequent genotypes in HSIL were HPV16 (34.3\%), HPV52 (15.0\%), and HPV58 (6.7\%) [21]. In this study, the order of top three frequent HPV types in HSIL was the same with that reported in the metaanalysis, but more HPV52 $(24.4 \%, 95 \% \mathrm{CI}=15.8 \%$ to $34.9 \%)$ and HPV58 $(14.0 \%, 95 \% \mathrm{CI}=7.4 \%$ to $23.1 \%)$ were detected in this study. Given that the PGMY-CHUV assay exhibits a higher sensitivity and specificity for detection of HPV52 and 58 in multiple infection specimens [13], the previous surveys may have underestimated the prevalence of these genotypes. This may be also true of the HPV type distribution in LSIL cases. The meta-analysis revealed that five most frequent HPV types in LSIL were HPV16 (13.8\%), HPV52 (11.1\%), HPV51 (9.8\%), HPV56 (9.0\%), and HPV58 (5.8\%) [21], but reported lower distribution rates for HPV52, 56, and 58, compared to those detected in this study: HPV52 $(16.4 \%, 95 \% \mathrm{CI}=10.6 \%$ to $23.8 \%)$, HPV56 $(14.9 \%$,

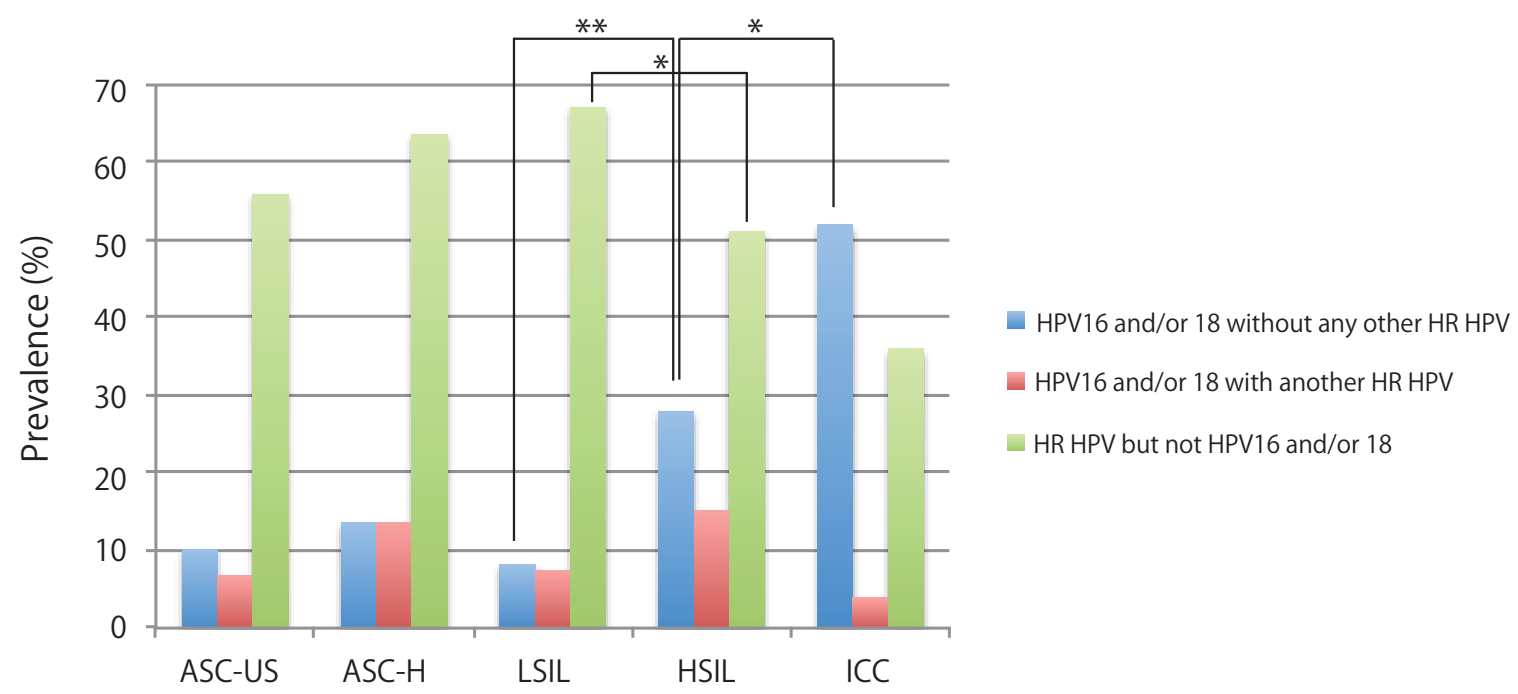

Fig. (3). Prevalence of HPV16 and/or 18 and other high-risk HPVs (alone or in mixed infections with HPV16 and/or 18). The percentages of cases are presented by each cervical abnormality grade. * and ** indicate $P<0.05$ and $P<0.001$, respectively, 
Table 3. Multiple Infections (MI) of HPV in Japanese Women with Abnormal Cervical Cytology

\begin{tabular}{|c|c|c|c|c|c|c|c|c|c|c|c|c|}
\hline & \multicolumn{2}{|c|}{ Total } & \multicolumn{2}{|c|}{ ASC-US } & \multicolumn{2}{|c|}{ ASC-H } & \multicolumn{2}{|c|}{ LSIL } & \multicolumn{2}{|c|}{ HSIL } & \multicolumn{2}{|c|}{ ICC } \\
\hline $\operatorname{HPV}(+)^{*}$ & \multicolumn{2}{|c|}{307} & \multicolumn{2}{|c|}{49} & \multicolumn{2}{|c|}{20} & \multicolumn{2}{|c|}{130} & \multicolumn{2}{|c|}{85} & \multicolumn{2}{|c|}{23} \\
\hline $\mathrm{MI}^{*}$ & \multicolumn{2}{|c|}{91} & \multicolumn{2}{|c|}{12} & \multicolumn{2}{|c|}{8} & \multicolumn{2}{|c|}{42} & \multicolumn{2}{|c|}{27} & \multicolumn{2}{|c|}{2} \\
\hline$\%{ }^{* *}$ & \multicolumn{2}{|c|}{29.6} & \multicolumn{2}{|c|}{24.5} & \multicolumn{2}{|c|}{40.0} & \multicolumn{2}{|c|}{32.3} & \multicolumn{2}{|c|}{31.8} & \multicolumn{2}{|c|}{8.7} \\
\hline & $\mathrm{n}^{*}$ & $\%^{* *}$ & $\mathbf{n}^{*}$ & $\%^{* *}$ & $\mathbf{n}^{*}$ & $\%^{* * *}$ & $n^{*}$ & $\%^{* *}$ & $\mathbf{n}^{*}$ & $\%^{* *}$ & $\mathrm{n}^{*}$ & $\%^{* *}$ \\
\hline 1 type & 216 & 70.4 & 37 & 75.5 & 12 & 60.0 & 88 & 67.7 & 58 & 68.2 & 21 & 91.3 \\
\hline 2 types & 62 & 20.2 & 8 & 16.3 & 7 & 35.0 & 28 & 21.5 & 17 & 20.0 & 2 & 8.7 \\
\hline 3 types & 22 & 7.2 & 2 & 4.1 & 1 & 5.0 & 11 & 8.5 & 8 & 9.4 & 0 & 0 \\
\hline 4 types & 6 & 2.0 & 2 & 4.1 & 0 & 0 & 3 & 2.3 & 1 & 1.2 & 0 & 0 \\
\hline 5 types & 1 & 0.3 & 0 & 0 & 0 & 0 & 0 & 0 & 1 & 1.2 & 0 & 0 \\
\hline \multicolumn{13}{|c|}{ MI of High-Risk HPVs } \\
\hline & $\mathbf{n}^{*}$ & $\%^{* * * *}$ & $\mathbf{n}^{*}$ & $\%^{* * *}$ & $\mathbf{n}^{*}$ & $\%^{* * * *}$ & $\mathbf{n}^{*}$ & $\%^{* * * *}$ & $\mathbf{n}^{*}$ & $\%^{* * * *}$ & $\mathbf{n}^{*}$ & $\%^{* * *}$ \\
\hline & 77 & 84.6 & 8 & 66.7 & 8 & 100 & 35 & 83.3 & 24 & 88.9 & 2 & 100 \\
\hline
\end{tabular}

*, Numbers of patients.

**, Percentages relative to the total number of HPV-positive patients in each cytological group.

***, Percentages relative to the total number of multiple-types infected patients in each cytological group.

$95 \% \mathrm{CI}=9.4 \%$ to $22.1 \%)$, and HPV58 $(17.2 \%, 95 \% \mathrm{CI}=$ $11.2 \%$ to $24.6 \%$ ).

In a meta-analysis using worldwide data on HPV type prevalence, a higher prevalence of HPV52 and 58 in HSIL and ICC was reported for East Asian countries, such as China, Taiwan, Japan, and Korea, compared to Europe, North America, and Africa [4,25], suggesting region-specific variation of HPV type prevalence in East Asia. Furthermore, a recent study by Takehara et al. [26] lends further support to the high prevalence of HPV52 and 58 in cervical intraepithelial lesions in Japanese women. Surprisingly, a recent clinical trial for the bivalent vaccine examining its cross-protective efficacy revealed negative vaccine efficacy for precancerous events associated with HPV52; vaccinated women developed slightly more HPV52-associated CIN2/3 lesions (excluding HPV16/18 co-infection) compared to the control women who received placebo [27]. Regarding an HPV genotyping method, it is worth noting that the InnoLiPA kit used in that trial was shown to be suboptimal for the detection of HPV52 in the global proficiency study of HPV genotyping [12], and thus may lead to underestimation of HPV52 in mixed infection samples, which indicates the necessity to confirm HPV52 prevalence by other typing methods or type-specific real-time PCR. Although the data are preliminary and will require further extensive evaluation, some potential for type-replacement, through which the protection of HPV16/18 infection by vaccines makes progression of HPV52-associated lesions more evident over time, warrants consideration, because high-risk HPVs not targeted by the vaccines tend to induce CIN3+ more slowly than HPV16/18 [28].

The HPV type distribution in CIN2/3 specimens of Japanese women was previously described in a study by Onuki et al. [15] as follows: three most frequent types were HPV16 (24.1\%), HPV52 (17.5\%), and HPV58 (10.7\%). The higher detection rate of HPV16 $(45.2 \%, 95 \% \mathrm{CI}=29.8 \%$ to $61.3 \%$ ) in CIN3 specimens in this study, as well as that reported in previous epidemiological studies [14,15,28], strongly indicates a higher risk of HPV16 infection for progression to high-grade intraepithelial neoplasia and/or invasive cervical cancer.

Although the number of cervical cancer samples in this study was relatively small $(n=25)$, the detection rates of HPV vaccine-targeted types were $40 \%$ for HPV 16 and $16 \%$ for HPV18, totaling $56 \%$ for both types, which lies in the range of 50 to $70 \%$ reported for cervical cancer in previous studies in Japan [7-9]. Further continuous investigation of the HPV type distribution in cervical cancer and its precursor lesions, such as CIN2/3 and adenocarcinoma in situ, in a central laboratory using a standardized genotyping assay will be required to monitor the efficacy of the current HPV vaccines, as well as to formulate a strategy for the development of the next generation HPV vaccines to meet the demand for protection from high-risk HPV infection other than HPV16/18 in each region, such as HPV52 and 58 in Japan and East Asia.

\section{ABBREVIATIONS}

$\mathrm{HPV}=$ Human papillomavirus

NILM = Negative for intraepithelial lesion or malignancy

ASC-US = Atypical squamous cells of undetermined significance

ASC-H = Atypical squamous cells, cannot exclude highgrade squamous intraepithelial lesion

LSIL = Low-grade squamous intraepithelial lesion

HSIL = High-grade squamous intraepithelial lesion

ICC = invasive cervical cancer 


$$
\begin{array}{ll}
\text { WHO } & =\text { World Health Organization } \\
\mathrm{CIN} & =\text { Cervical intraepithelial neoplasia } \\
\mathrm{SCC} & =\text { Squamous cell carcinoma } \\
\mathrm{CHUV} & =\text { Centre Hospitalier Universitaire Vaudois }
\end{array}
$$

\section{CONFLICT OF INTEREST}

The authors confirm that this article content has no conflict of interest.

\section{ACKNOWLEDGEMENTS}

We thank the WHO HPV LabNet European Reference Laboratory, Centre Hospitalier Universitaire Vaudois (CHUV) in Lausanne, Switzerland, for technical support of introduction of the PGMY-CHUV assay into our laboratory.

\section{FUNDING}

This work received financial support from the Ministry of Health, Labor and Welfare in Japan.

\section{REFERENCES}

[1] zur Hausen H, Papillomaviruses and cancer: from basic studies to clinical application. Nat Rev Cancer 2002; (2): 342-50.

[2] de Villiers EM, Fauquet C, Broker TR, Bernard HU, zur Hausen H. Classification of papillomaviruses. Virology 2004; 324: 17-27.

[3] Munoz N, Bosch FX, de Sanjose S, et al. Epidemiologic classification of human papillomavirus types associated with cervical cancer. N Engl J Med 2003; 348: 518-27.

[4] Clifford GM, Smith JS, Aguado T, Franceschi S. Comparison of HPV type distribution in high-grade cervical lesions and cervical cancer: a meta-analysis. Br J Cancer 2003; 89: 101-5.

[5] The FUTURE II Study Group Quadrivalent vaccine against human papillomavirus to prevent high-grade cervical lesions. N Engl J Med 2007; 356: 1915-27.

[6] Paavonen J, Naud P, Salmeron J, et al. Efficacy of human papillomavirus (HPV)-16/18 AS04-adjuvanted vaccine against cervical infection and precancer caused by oncogenic HPV types (PATRICIA): final analysis of a double-blind, randomised study in young women. Lancet 2009; 374: 301-14.

[7] Asato T, Maehama T, Nagai Y, et al. A large case-control study of cervical cancer risk associated with human papillomavirus infection in Japan, by nucleotide sequencing-based genotyping. $\mathrm{J}$ Infect Dis 2004; 189: 1829-32.

[8] Nakagawa S, Yoshikawa H, Onda $\mathrm{T}$, et al. Type of human papillomavirus is related to clinical features of cervical carcinoma. Cancer 1996; (78): 1935-41.

[9] Sasagawa T, Basha W, Yamazaki H, Inoue M. High-risk and multiple human papillomavirus infections associated with cervical abnormalities in Japanese women. Cancer Epidemiol Biomarkers Prev 2001; (10): 45-52.

[10] Estrade C, Menoud PA, Nardelli-Haefliger D, Sahli R. Validation of a low-cost human papillomavirus genotyping assay based on PGMY PCR and reverse blotting hybridization with reusable membranes. J Clin Microbiol 2011; 49: 3474-81.

[11] World Health Organization human papillomavirus laboratory manuaL, $1^{\text {st }}$ ed. Available at: http://wwwwhoint/immunization/do cume nts/WHO_IVB 2009;_1012/en/ (WNO/IVB/10.12) WHO, Geneva (Switzerland).

[12] Eklund C, Zhou T, Dillner J. Global proficiency study of human papillomavirus genotyping. J Clin Microbiol 2010; 48: 4147-55.

[13] Mori S, Nakao S, Kukimoto I, et al. Biased amplification of human papillomavirus DNA in specimens containing multiple human papillomavirus types by PCR with consensus primers. Cancer Sci 2011; 102: 1223-7.

[14] Matsumoto K, Oki A, Furuta R, et al. Predicting the progression of cervical precursor lesions by human papillomavirus genotyping: a prospective cohort study. Int J Cancer 2011; 128: 2898-910.

[15] Onuki M, Matsumoto K, Satoh T, et al. Human papillomavirus infections among Japanese women: age-related prevalence and type-specific risk for cervical cancer. Cancer Sci 2009; 100: 13126.

[16] Maehama T, Asato T, Kanazawa K. Prevalence of HPV infection in cervical cytology-normal women in Okinawa, Japan, as determined by a polymerase chain reaction. Int $\mathrm{J}$ Gynaecol Obstet 2000; 69: 175-6.

[17] Yoshikawa H, Nagata C, Noda K, et al. Human papillomavirus infection and other risk factors for cervical intraepithelial neoplasia in Japan. Br J Cancer 1999; 80: 621-4.

[18] Yoshikawa H, Kawana T, Kitagawa K, Mizuno M, Yoshikura H, et al. Detection and typing of multiple genital human papillomaviruses by DNA amplification with consensus primers. Jpn J Cancer Res 1991; 82: 524-31.

[19] Yoshida T, Fukuda T, Sano T, et al. Usefulness of liquid-based cytology specimens for the immunocytochemical study of p16 expression and human papillomavirus testing: a comparative study using simultaneously sampled histology materials. Cancer 2004; 102: $100-8$.

[20] Nagai Y, Maehama T, Asato T, Kanazawa K. Persistence of human papillomavirus infection after therapeutic conization for CIN 3: is it an alarm for disease recurrence? Gynecol Oncol 2000; 79: 294-9.

[21] Miura S, Matsumoto K, Oki A, et al. Do we need a different strategy for HPV screening and vaccination in East Asia? Int $\mathrm{J}$ Cancer 2006; 119: 2713-15.

[22] Castle PE, Schiffman M, Gravitt PE, et al. Comparisons of HPV DNA detection by MY09/11 PCR methods. J Med Virol 2002; 68: 417-23.

[23] Castle PE, Solomon D, Schiffman M, Wheeler CM. Human papillomavirus type 16 infections and 2-year absolute risk of cervical precancer in women with equivocal or mild cytologic abnormalities. J Natl Cancer Inst 2005; 97: 1066-71.

[24] Brismar-Wendel S, Froberg M, Hjerpe A, Andersson S, Johansson B. Age-specific prevalence of HPV genotypes in cervical cytology samples with equivocal or low-grade lesions. Br J Cancer 2009; 101: 511-7.

[25] Smith JS, Lindsay L, Hoots B, et al. Human papillomavirus type distribution in invasive cervical cancer and high-grade cervical lesions: a meta-analysis update. Int J Cancer 2007; 121: 621-32.

[26] Takehara K, Toda T, Nishimura T, et al. Human papillomavirus types 52 and 58 are prevalent in uterine cervical squamous lesions from Japanese women. Patholog Res Int 2011; 2011: 246936.

[27] Wheeler CM, Castellsague X, Garland SM, et al. Cross-protective efficacy of HPV-16/18 AS04-adjuvanted vaccine against cervical infection and precancer caused by non-vaccine oncogenic HPV types: 4-year end-of-study analysis of the randomised, double-blind PATRICIA trial. Lancet Oncol 2012; 13: 100-10.

[28] Kjaer SK, Frederiksen K, Munk C, Iftner T. Long-term absolute risk of cervical intraepithelial neoplasia grade 3 or worse following human papillomavirus infection: role of persistence. J Natl Cancer Inst 2010; 102: 1478-88. 